Published in final edited form as:

Gynecol Oncol. 2013 February ; 128(2): 155-159. doi:10.1016/j.ygyno.2012.11.034.

\title{
Is Bilateral Lymphadenectomy For Midline Squamous Carcinoma Of The Vulva Always Necessary? An Analysis From Gynecologic Oncology Group (GOG) 173
}

\author{
Robert L. Coleman, MD1, Shamshad Ali, MS ${ }^{2}$, Charles F. Levenback, MD ${ }^{1}$, Michael A. Gold, \\ $M^{3,{ }^{*}}$, Jeffrey M. Fowler, MD ${ }^{4}$, Patricia L. Judson, MD $^{5}$, Maria C. Bell, MD ${ }^{6}$, Koen De Geest, \\ MD ${ }^{7}$, Nick M. Spirtos, MD ${ }^{8}$, Ronald K. Potkul, MD ${ }^{9}$, Mario M. Leitao Jr, MD ${ }^{10}$, Jamie N. \\ Bakkum-Gamez, MD ${ }^{11}$, Emma C. Rossi, MD ${ }^{12}$, Samuel S. Lentz, MD ${ }^{13}$, James J. Burke II, \\ MD $^{14}$, Linda Van Le, MD ${ }^{15}$, and Cornelia L. Trimble, MD ${ }^{16}$ \\ ${ }^{1}$ The University of Texas MD Anderson Cancer Center, Houston, TX 77030 \\ ${ }^{2}$ Gynecologic Oncology Group Statistical \& Data Center; Roswell Park Cancer Institute, Buffalo, \\ NY 14263 \\ ${ }^{3}$ University of Oklahoma, Oklahoma City, OK 73190 \\ ${ }^{4}$ The Ohio State University; Columbus Cancer Council, Hilliard, OH 43026 \\ ${ }^{5}$ Moffitt Cancer Center, Tampa, FL 33612 \\ ${ }^{6}$ Sanford University of South Dakota Medical Center, Sioux Falls, SD 57105 \\ ${ }^{7}$ University of lowa Hospitals and Clinics, lowa City, IA 52242 \\ 8Women's Cancer Center of Nevada, Las Vegas, NV 89169 \\ ${ }^{9}$ Loyola University Medical Center, Maywood, IL 60153 \\ ${ }^{10}$ Memorial Sloan-Kettering Cancer Center, New York, NY 10021 \\ ${ }^{11}$ Mayo Clinic, Rochester, MN 55905 \\ ${ }^{12}$ Indiana University Medical Center, Indianapolis, IN 46202 \\ ${ }^{13}$ Wake Forest University, Winston-Salem, NC 27157 \\ ${ }^{14}$ Mercer University School of Medicine, Savannah Campus, Savannah, GA 31404 \\ ${ }^{15}$ University of North Carolina, Chapel Hill, NC 27599 \\ ${ }^{16}$ Johns Hopkins University School of Medicine; Baltimore, MD 21287
}

\section{Abstract}

(C) 2012 Elsevier Inc. All rights reserved.

Corresponding Author: Robert L. Coleman, MD, University of Texas, M.D. Anderson Cancer Center, 1155 Herman Pressler Dr., CPB 6.3271, Houston, TX 77030, Ph: 713-745-3357, Fax: 713-792-7586, rcoleman@ mdanderson.org.

Michael Gold is currently affiliated with Vanderbilt University; Nashville, TN 37232

Publisher's Disclaimer: This is a PDF file of an unedited manuscript that has been accepted for publication. As a service to our customers we are providing this early version of the manuscript. The manuscript will undergo copyediting, typesetting, and review of the resulting proof before it is published in its final citable form. Please note that during the production process errors may be discovered which could affect the content, and all legal disclaimers that apply to the journal pertain.

CONFLICT OF INTEREST

Dr. Linda Van Le is on the Speaker's Bureau for Eisai and the Advisory Board for Biologics, Inc. All other co-authors have no conflicts of interest to declare. 
Objective-To determine which patients with near midline lesions may safely undergo unilateral groin dissection based on clinical exam and lymphoscintigraphy (LSG) results.

Methods-Patients participating in GOG-173 underwent sentinel lymph node (SLN) localization with blue dye, and radiocolloid with optional LSG before definitive inguinal-femoral lymphadenectomy (LND). This analysis interrogates the reliability of LSG alone relative to primary tumor location in those patients who had an interpretable LSG and at least one SLN identified. Primary tumor location was categorized as lateral ( $>2 \mathrm{~cm}$ from midline), midline, or lateral ambiguous (LA) if located within $2 \mathrm{~cm}$., but not involving the midline.

Results-Two-hundred-thirty-four patients met eligibility criteria. Sixty-four had lateral lesions, and underwent unilateral LND. All patients with LA $(\mathrm{N}=65)$ and midline $(\mathrm{N}=105)$ tumors underwent bilateral LND. Bilateral drainage by LSG was identified in 14/64 (22\%) patients with lateral tumors, 38/65 (58\%) with LA tumors and in 73/105 (70\%) with midline tumors. At mapping, no SLNs were found in contralateral groins among those patients with LA and midline tumors who had unilateral-only LSGs. However, in these patients groin metastases were found in $4 / 32$ patients with midline tumors undergoing contralateral dissection; none were found in 27 patients with LA tumors.

Conclusion-The likelihood of detectable bilateral drainage using preoperative LSG decreases as a function of distance from midline. Patients with LA primaries and unilateral drainage on LSG may safely undergo unilateral SLN.

\section{Keywords}

Vulva cancer; sentinel node; lymphadenectomy; lymphoscintigraphy; sentinel node mapping; radiocolloid; vital blue dye

\section{INTRODUCTION}

Primary carcinoma of the vulva is a rare gynecological malignancy afflicting less than 5000 women in the US annually [1]. Limited stage disease, which accounts for about half of these cases, is primarily approached by surgical extirpation and lymphadenectomy. Refinements in surgical technique and utility of appropriate adjuvant therapy have improved morbidity and mortality from this disease [2-5]. However, chronic lower extremity edema - reported in more than $30 \%$ of patients after groin lymphadenectomy - is a common post-operative morbidity, which is difficult to manage and significantly impairs quality of life. (ref: Gaarenstroom KN, et al., Int J Gynecol Cancer 13:522-527, 2003; DeHullu JA, et al., J psychosom Obstet Gynecol 22:199-203, 2001). Therefore, the ability to identify a subset of patients who might be able to undergo a less radical primary procedure would be of great clinical benefit.

The clinical observation that lymphatic drainage from the vulva to the groin proceeds in predictable fashion relative to the primary tumor location, has prompted the evaluation of sentinel lymph node (SLN) assessment as an alternative to full inguinofemoral lymphadenectomy. Currently, the most commonly used technique to identify sentinel nodes uses intradermal injection of vital dye, followed by direct, open visualization and limited groin exploration and node resection [6]. Outcomes data from two large observational studies of SLN biopsy in women with limited stage disease have entered the public domain [7,8]. While the two trials differed with respect to eligibility, mapping technique, utilization of skill verification, primary endpoint and recommendation for groin dissection in the presence of a metastatic SLN, they produced remarkably consistent results: the SLN biopsy technique is feasible and is associated with a false negative predictive value of $3 \%$ or less in 
appropriately selected patients (T1 or $\mathrm{T} 2$ tumors less than $4 \mathrm{~cm}$ and clinically negative groins).

Surgical recommendations regarding unilateral or bilateral groin dissection in patients with clinically non-suspicious groins (N0, N1) are generally dictated by the location of vulvar primary. Lesions more than 1-2 cm lateral to the midline structures are approached with ipsilateral dissection; those within this boundary are approached with bilateral dissection. The latter is recommended based on the increasing probability of contralateral flow when lesions approach dermal lymphatics that may cross the midline. This concept is further supported by studies demonstrating that contralateral groin failure in patients undergoing ipsilateral groin dissection for these "lateralized" primaries is very low $(<3 \%)[9,10]$. Since nodal metastases occur in the minority of women with early stage (T2) vulvar cancer (20 to $30 \%$ ), and SLN localization techniques provide an opportunity to examine "real-time" anatomical lymphatic drainage in these patients, we hypothesized that preoperative lymphoscintigraphy could be useful in guiding groin dissection recommendations, particularly for lesions within $2 \mathrm{~cm}$, but not involving the midline. Herein, we report the analysis of lymphoscintigraphy performed in patients participating in Gynecologic Oncology Group (GOG) Protocol 173.

\section{METHODS}

\section{Patients}

The study design, preoperative and intraoperative testing and surgical procedures for this study have been previously presented [8]. Briefly, women with biopsy confirmed squamous cell carcinoma with T2 lesions less than $6 \mathrm{~cm}$ in greatest dimension and with clinically negative (N0 or N1) groins were eligible to participate. Initially, patients meeting eligibility were required only to have SLN localization via blue dye (20\% of the sample population). However, the study was amended two years following activation to require injection of radiocolloid, with optional lymphoscintigraphy. Lymphoscintigraphy was performed prior to surgery, following the intradermal injection of 0.5 to $1.0 \mathrm{ml}$ of radiolabeled Tc99 microsulfur colloid at the leading border of the primary tumor (the border closest to the groin). Injection on both sides of the tumor was required for patients with midline tumors. Anatomical markers were placed on the symphysis pubis and anterior superior iliac spines, which was followed by an immediately scan. The skin over a candidate SLN was marked with an indelible marker. Transmission scans showing the outline of the body were preferred, if possible. Other radionuclides were allowed as substitutions if Tc99 microsulfur colloid was not available. EMLA cream was to be applied 30-60 minutes prior to the injection of the radionuclide as a local anesthetic. These methods increased the frequency of SLN detection. All enrolled patients underwent inguinofemoral lymphadenectomy following SLN localization. Patients with clearly lateralized tumors underwent unilateral lymphadenectomy. Those with primary tumors within $2 \mathrm{~cm}$ of the midline underwent bilateral groin dissection. For this analysis, primary vulvar lesions were defined as lateral (more than $2 \mathrm{~cm}$ from the midline), midline (tumor involving the midline) or lateral/ ambiguous (LA) (tumor not involving the midline but not meeting the criteria for a lateral primary), based on hand-drawn pictorial representations of the primary tumor, tumor description from operative notes and/or final pathology reports of the excised specimen.

\section{Study Methodology}

This prospective multi-institutional trial was conducted in 47 centers with no specific skill verification and averaged about six cases per site during the enrollment period (December 1999 to December 2009). The primary statistical outcome measure of feasibility was the false negative predictive value, which considered the rate of false negative identification 
(SLN histologically negative for metastases but with histologically metastatic non-sentinel groin nodes) in affected groins and the true negative rate in unaffected dissected groins. The staged enrollment design was based on the number of patients with histologically confirmed groin node metastases maintaining a sensitivity of greater than $87 \%$ and a false negative predictive value of under $5 \%$.

GOG 173 accrued 515 assessable patients; the metastatic node rate was $32 \%$.

\section{Statistical Methods}

The analysis set is comprised of eligible GOG 0173 patients with interpretable LSG (N = 234).

Pearson chi-square test was employed to assess statistical significance of observed associations and/or trends among nominal variables [11]. Bar graphs and contingency tables were used to summarize and display pertinent results. Estimated proportions are reported with $95 \%$ confidence limits, where appropriate. Nominal data comparisons are made using Chi Square test, including the analysis of trend for lateralized lesions and bilateral lymphoscintigram uptake.

\section{RESULTS}

Figure 1 demonstrates how the 234 eligible candidates for this analysis were identified. While 84 patients did not have a lymphoscintigram performed, the largest contributors to inevaluability ( $\mathrm{N}=126)$ were: uninterpretable study, study not submitted for review, if done, or uniformative study due to no activity in the groin. Figure 2 demonstrates that the rate of bilateral drainage on preoperative lymphoscintigram is significantly related to the location of the primary tumor.

\section{Lateral Primary Tumors}

The relationship of preoperative lymphoscintigraphy, SLN localization, and final sentinel and non-SLN groin histology in subjects with lateral primary lesions $(n=64)$ is presented in Table 1. These patients all underwent a unilateral groin dissection on the ipsilateral side. Lymphoscintigraphy identified drainage on only the ipsilateral side in 50/64 (78\%), and bilateral drainage in 14/64 (22\%). At surgery, SLNs were identified in all of the dissected ipsilateral groins. Of these SLNs, metastatic disease was identified in 11/64 (17\%). Three additional patients with pathologically negative SLNs were found with metastatic disease in the excised non-sentinel nodal specimens (false negative SLNs).

\section{Midline Primary Tumors}

One-hundred-five patients had midline tumors, all of which invaded or occurred on midline vulvar anatomical structures; all underwent bilateral groin dissections. The pattern of lymphatic drainage identified by LSG, i.e. unilateral vs bilateral, was concordant with patterns identified using dye/radiocolloid in 95/105 (90\%) of cases. Unilateral drainage by LSG was demonstrated in 32/105 (31\%) patients. A SLN was found in all 32 of these women; interestingly, no SLN was identified in the contralateral groin where the LSG demonstrated no flow. In this cohort, metastatic nodal disease was found in 16 patients (50\%); one patient had a pathologically negative SLN but was found with metastatic disease in the residual groin nodal tissue ( $6 \%$ false negative rate). Among the 73 patients with bilateral drainage detected by lymphoscintigraphy, SLNs were identified by intraoperative mapping bilaterally in 63 patients (86\%), unilaterally in $6(8 \%)$ and not identified in $4(5 \%)$ (Table 2). Among the 63 patients with SLN found in both groins, 18 had metastatic disease; in three patients a pathologically unaffected SLN was identified in the presence of groin 
metastases in the excised residual nodal tissue ( $14 \%$ false negative rate). Among the six patients with bilateral flow by LSG but unilaterally identified SLNs, no metastatic disease was identified in either the SLN or the residual groin nodal tissue. Among the four patients without a SLN identified, two were found with groin metastases. The overall rate of metastatic disease for women with midline tumors was $41 \%$ (43/105 patients). Thus, although data from this subset supports the accuracy of LSG, it does not clearly identify a subset of patients in whom the technology could be used to restrict the scope of the initial operative procedure.

\section{Midline Primary Tumors Not Invading Midline Structures (Lateral/Ambiguous)}

-There were 65 patients with lateral/ambiguous primary tumors. Preoperative LSG showed unilateral drainage in 27/65 (42\%) of subjects all of whom had a SLN identified, ipsilaterally. Among these patients $10(37 \%)$ were found with metastatic groin pathology all in ipsilateral groins. The SLN had metastatic disease in nine of these 10 cases ( 1 false negative). No SLNs were identified at mapping on the side that had no detectable drainage by LSG, and there were no positive nodes on the contralateral side in these fully dissected groins. Among patients with bilateral LSG drainage, SLNs were found on one side in 14/38 (38\%) and on both sides in $23 / 38$ (62\%) of patients. The node positivity rate was $40 \%$ in this group and there was only one false-negative case.

\section{DISCUSSION}

In this study of lymphoscintigraphy performed as specified in GOG 173, we documented the following: primary squamous cell tumors between 2 and $6 \mathrm{~cm}$ in greatest dimension can have variable and ambiguous lymphatic drainage; that this drainage is related to primary location relative to the midline; that preoperative assessment with lymphoscintigraphy may inform the surgical approach in patients with lateral/ambiguous primary tumors. This latter hypothesis is important because, if validated, it would impact a significant number of patients (approximately $16 \%$ or $27 / 170$ ), who by the nature of their primary lesion would otherwise undergo unnecessary SLN biopsy and/or bilateral groin dissection. This is because tumors within $2 \mathrm{~cm}$ of the midline are surgically managed as "midline" lesions with the contemporary standard surgical approach of bilateral groin node dissection.

It should be cautioned, though, that lack of bilateral drainage in true midline lesions could not be used with the same degree of confidence. In the current analysis, $30 \%$ of women with tumor invading or crossing the midline had unilateral drainage by lymphoscintigraphy. In these cases, although no SLNs were identified, four patients had metastatic disease in the contralateral groin.

Historically, LSG was first employed in patients with truncal melanoma to help focus locations where lymph node dissection should be performed. In these cases, potential preferred drainage sites could be in the axillae, groin or both [12]. We wished to test the hypothesis that lymphoscintigraphy could be similarly informative in ambiguously located primary squamous vulva carcinomas.

We recognize the limitations of the current study, which was conducted to raise important new hypotheses with regard to the SLN procedure in the care of women with primary vulvar cancer. One significant limitation is the fact that lymphoscintigraphy was collected in less than half of enrolled subjects. This might overestimate the proportion of informative cases in each of the categories reviewed. However, the node-positive rate and negative predictive value for the current study population was similar to that reported in GOG 173. Another limitation is the lack of follow-up data on adjuvant treatment and recurrence rates. It is appropriately inferred that false negative SLN assessment would lead to higher rates of groin 
recurrence relative to those with full groin dissection, particularly if untreated adjuvantly. However, in GOG 173 all patients undergoing SLN localization, whether or not a SLN was identified, were to undergo a full inguinal-femoral lymphadenectomy in that groin. It is therefore not possible to estimate the impact of SLN biopsy alone in this sample. Similarly, the absence of recurrence information also makes the evaluation of the cases of lateral primary lesion with bilateral drainage on lymphoscintigraphy (in whom only unilateral dissections were done) impossible. Ultimate recurrence in these undissected groins would strengthen the predictive power of the study with regard to preoperative surgical treatment planning. Fortunately, an ongoing multi-cooperative group trial (GROINS-VII/GOG-270, NCT01500512) is evaluating a cohort of SLN negative patients who are managed without groin dissection or adjuvant treatment for the primary endpoint of groin recurrence.

The seminal work by US and European investigators demonstrating the feasibility of performing SLN evaluation for women with early stage vulvar cancer is continuing to investigate surgical modification in this disease. The morbidity from routine lymphadenectomy, particularly in combination with radiation or chemoradiation, is not trivial and is a major cause of reduction in quality of life for these women [13]. The GROINS-VII/GOG270 protocol will additionally evaluate the role of SLN biopsy and directed radiation for small nodal metastases (less than $2 \mathrm{~mm}$ ). The absence of full lymphadenectomy is a distinct departure from current treatment recommendations, but has the potential to improve outcomes and quality of life if proved feasible.

\section{Acknowledgments}

This study was supported by National Cancer Institute grants to the Gynecologic Oncology Group Administrative Office (CA 27469), the Gynecologic Oncology Group Statistical and Data Center (CA 37517). The following institutions participated in this study: Roswell Park Cancer Institute, University of Alabama at Birmingham, Abington Memorial Hospital, Walter Reed Army Medical Center, University of Minnesota Medical School, University of Mississippi Medical Center, Colorado Gynecologic Oncology Group P.C., University of Pennsylvania Cancer Center, Milton S. Hershey Medical Center, University of Cincinnati, University of North Carolina School of Medicine, University of Iowa Hospitals and Clinics, University of Texas Southwestern Medical Center at Dallas, Indiana University School of Medicine, Wake Forest University School of Medicine, University of California Medical Center at Irvine, Tufts-New England Medical Center, Rush-Presbyterian-St. Luke's Medical Center, Magee Women's Hospital, The Cleveland Clinic Foundation, Washington University School of Medicine, Memorial Sloan-Kettering Cancer Center, Columbus Cancer Council, MD Anderson Cancer Center, Fox Chase Cancer Center, Women's Cancer Center, University of Oklahoma, University of Virginia Health Sciences Center, University of Chicago, Mayo Clinic, Case Western Reserve University, Tampa Bay Cancer Consortium, Gynecologic Oncology Network, Fletcher Allen Health Care, University of Wisconsin Hospital, The Hospital of Central Connecticut, Georgia CORE Consortium, and Community Clinical Oncology Program.

\section{REFERENCES}

1. Jemal A, Bray F, Center MM, Ferlay J, Ward E, Forman D. Global cancer statistics. CA Cancer J Clin. 2011; 61:69-90. [PubMed: 21296855]

2. Helm CW, Hatch K, Austin JM, Partridge EE, Soong SJ, Elder JE, et al. A matched comparison of single and triple incision techniques for the surgical treatment of carcinoma of the vulva. Gynecol Oncol. 1992; 46:150-156. [PubMed: 1500015]

3. Burke TW, Stringer CA, Gershenson DM, Edwards CL, Morris M, Wharton JT. Radical wide excision and selective inguinal node dissection for squamous cell carcinoma of the vulva. Gynecol Oncol. 1990; 38:328-332. [PubMed: 2227543]

4. Berman ML, Soper JT, Creasman WT, Olt GT, DiSaia PJ. Conservative surgical management of superficially invasive stage I vulvar carcinoma. Gynecol Oncol. 1989; 35:352-357. [PubMed: 2599471]

5. Lanza A, Valli M, Caldarola B, et al. Radical vulvectomy and inguinal lymphadenectomy versus inguino-pelvic lymphadenectomy combined with radical vulvectomy and the role of radiotherapy. Eur J Gynaec Oncol. 1988; 9:67-73. 
6. Alex JC, Weaver DL, Fairbank JT, Rankin BS, Krag DN. Gamma-probe-guided lymph node localization in malignant melanoma. Surg Oncol. 1993; 2:303-308. [PubMed: 8305972]

7. Van der Zee AG, Oonk MH, De Hullu JA, Ansink AC, Vergote I, Verheijen RH, et al. Sentinel node dissection is safe in the treatment of early-stage vulvar cancer. J Clin Oncol. 2008; 26:884889. [PubMed: 18281661]

8. Levenback CF, Ali S, Coleman RL, Gold MA, Fowler JM, Judson PL, et al. Lymphatic Mapping and Sentinel Lymph Node Biopsy in Women with Squamous Carcinoma of the Vulva: A Gynecologic Oncology Group Study. J Clin Oncol. 2012 [Epub ahead of print].

9. Burke TW, Levenback C, Coleman RL, Morris M, Silva EG, Gershenson DM. Surgical therapy of $\mathrm{T} 1$ and T2 vulvar carcinoma: further experience with radical wide excision and selective inguinal lymphadenectomy. Gynecol Oncol. 1995; 57:215-220. [PubMed: 7729737]

10. Stehman FB, Bundy BN, Dvoretsky PM, Creasman WT. Early stage I carcinoma of the vulva treated with ipsilateral superficial inguinal lymphadenectomy and modified radical hemivulvectomy: a prospective study of the Gynecologic Oncology Group. Obstet Gynecol. 1992; 79:490-497. [PubMed: 1553164]

11. Agresti, A. An Introduction to Categorical Data Analysis. Second Edition. New York: John Wiley \& Sons; 2007.

12. Morton DL, Wen DR, Wong JH, Economou JS, Cagle LA, Storm FK, et al. Technical details of intraoperative lymphatic mapping for early stage melanoma. Arch Surg. 1992; 127:392-399. [PubMed: 1558490]

13. Barton DP. The prevention and management of treatment related morbidity in vulval cancer. Best Pract Res Clin Obstet Gynaecol. 2003; 17:683-701. [PubMed: 12965139] 


\section{HIGHLIGHTS}

- Lymphoscintigraphy can serve as a useful adjuvant tool in sentinel lymph node biopsy planning

- Primary lateral ambiguous (LA) vulvar cancers have variable lymphatic drainage including exclusively ipsilateral groin localization by lymphoscintigraphy

- LA tumors with unilateral LSG drainage are at low risk for contralateral nodal metastases and may avoid routine inguinal-femoral lymphadenectomy 


\section{5 patients}
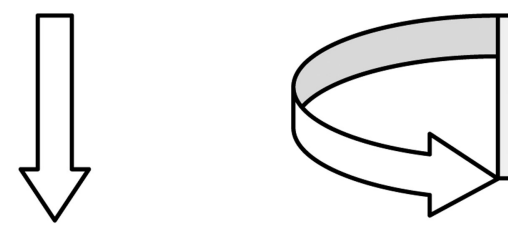

Excluded by centralized GOG modality reviews (56)

\section{9 patients}
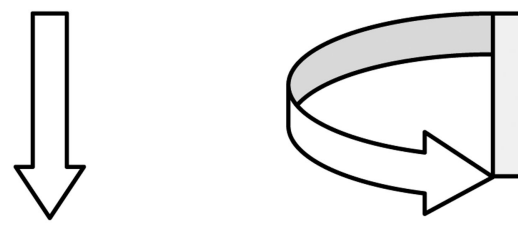

Either LN sampling or SLN mapping procedure not done (7)

\section{2 patients}
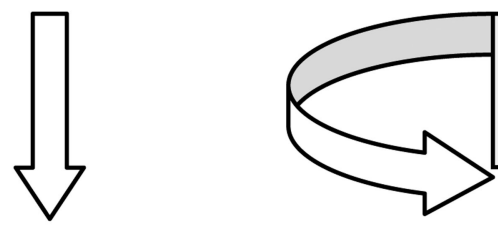

LSG not performed (92)

\section{0 patients}
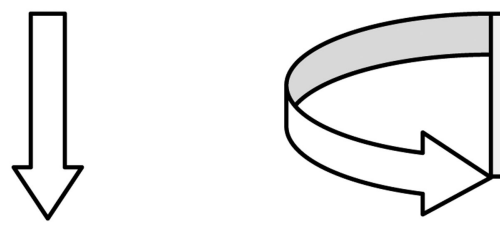

LSG report not submitted (97), LSG not interpretable (15), no SLN on image (14)

\section{4 patients}

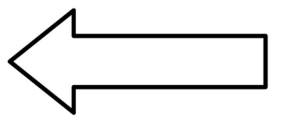

Evaluable for this project

Figure 1. Consort diagram

CONSORT diagram demonstrating patient exclusions producing the study cohort of 234 . 


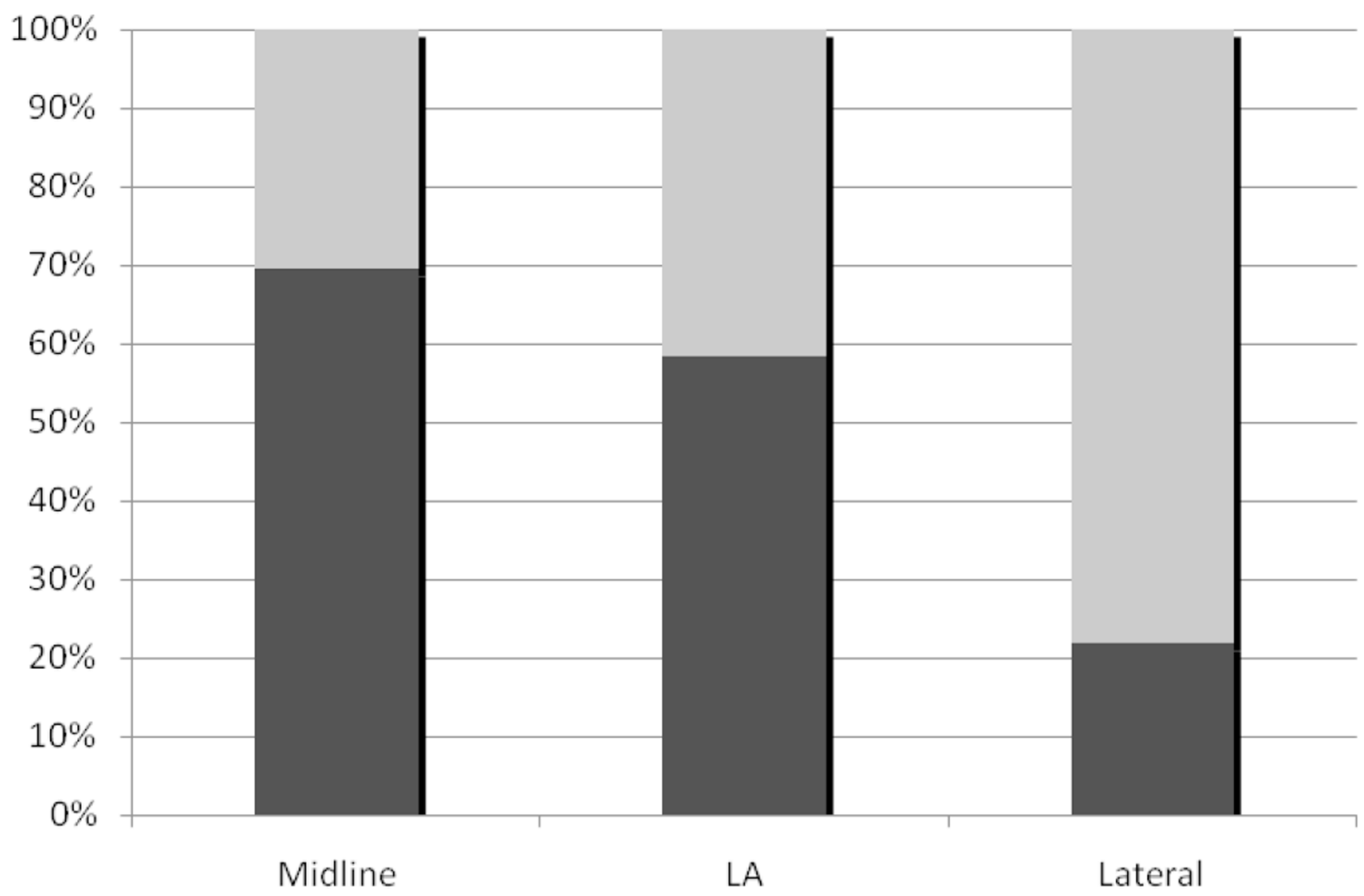

Unilateral

Bilateral

Figure 2. Distribution of unilateral/bilateral localization by Lymphoscintography

Frequency of bilateral drainage by lymphoscintigraphy relative to the location of the primary lesion as assessed by hand-drawn schematics, and operative or pathological reports. There is significant reduction in bilateral drainage as the primary lesion moves lateral (midline: $70 \%$, lateral/ambiguous: $58 \%$, lateral: $22 \%$ ). However, we observed that more than one in five patients with lateralized primary tumors (> 2 centimeters from the midline) had bilateral drainage on lymphoscintigraphy. 


\section{Table 1}

Relationship of lymphoscintigraphy drainage to sentinel lymph node localization and final pathology in lateral primary tumors (T2, less than 6 centimeters in greatest diameter, more than 2 centimeters from midline). All patients underwent unilateral inguinal-femoral lymphadenectomy.

\begin{tabular}{|c|c|c|c|}
\hline $\begin{array}{c}\text { LATERAL } \\
\text { PRIMARY }\end{array}$ & Ipsilateral & Contralateral & Total (\%) \\
\hline $\begin{array}{c}\text { LSG } \\
\text { drainage }\end{array}$ & $50(78 \%)$ & $14(22 \%)$ & 64 \\
\hline SLN Found & \multicolumn{2}{|c|}{$64(100 \%)$} & 64 \\
\hline $\begin{array}{c}\text { SLN } \\
\text { Pathology } \\
(+)\end{array}$ & 11 & $11(17 \%)$ \\
\hline $\begin{array}{c}\text { Groin } \\
\text { Pathology } \\
(+)\end{array}$ & (in patients with negative SLN) & $\begin{array}{c}\text { False Negative } \\
\text { (+14 (21\%) }\end{array}$ \\
\hline $\begin{array}{c}\text { Total Node } \\
(+)\end{array}$ & 14 & $22 \%$ \\
\hline
\end{tabular}




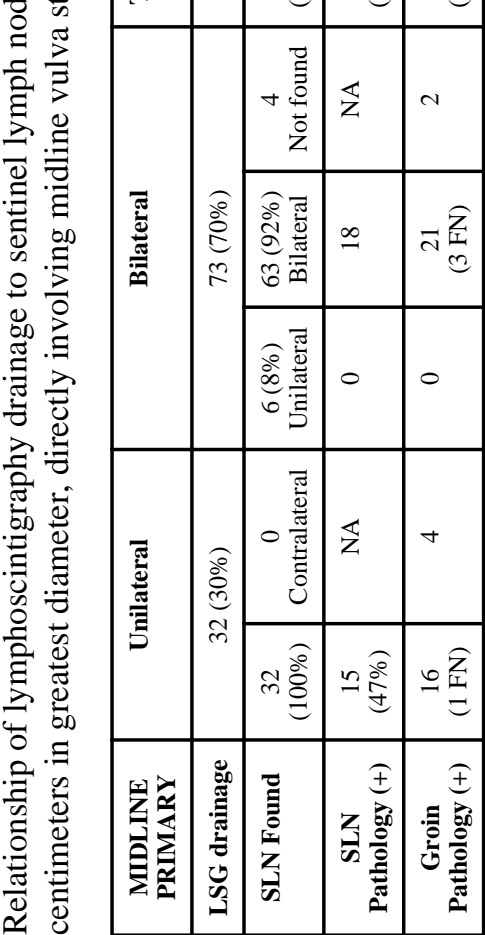




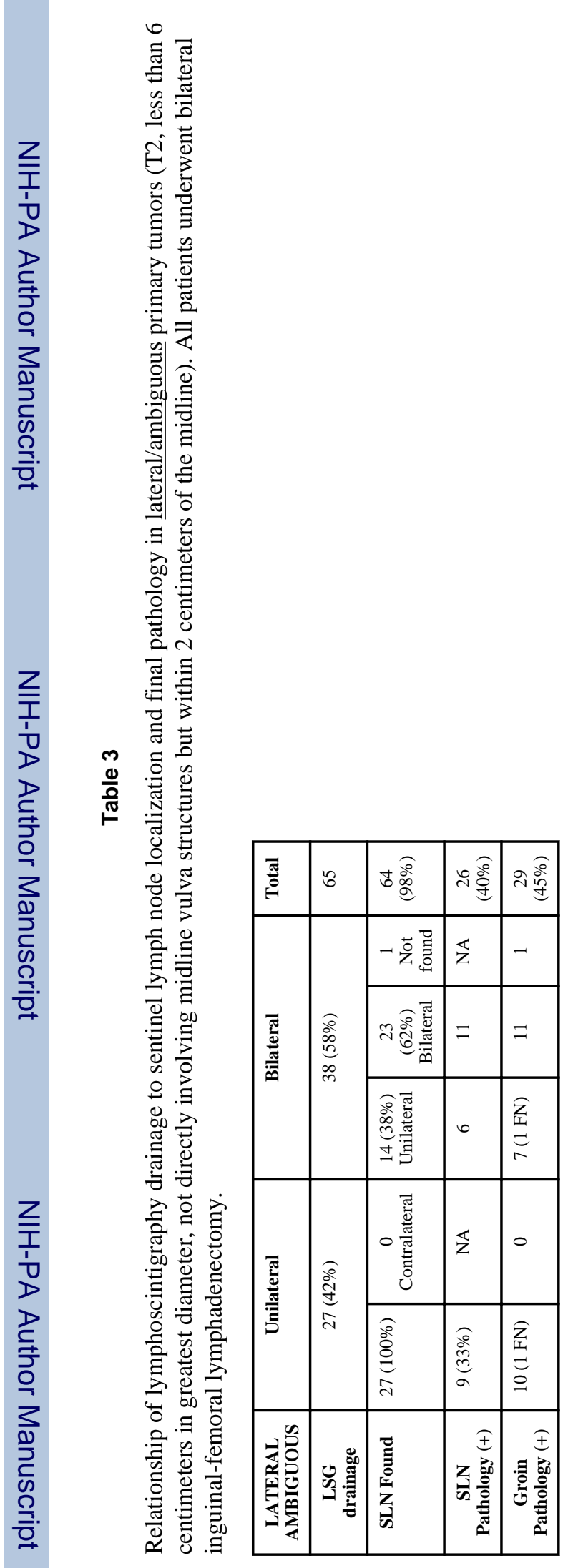

Gynecol Oncol. Author manuscript; available in PMC 2014 February 01. 